\title{
Anti-GITR Agonistic Monoclonal Antibody INCAGN01876
}

National Cancer Institute

\section{Source}

National Cancer Institute. Anti-GITR Agonistic Monoclonal Antibody INCAGN01876. NCI

Thesaurus. Code C126643.

An anti-human glucocorticoid-induced tumor necrosis factor receptor (tumor necrosis factor superfamily, member 18; TNFRSF18; GITR; CD357) agonistic humanized monoclonal antibody, with potential immune checkpoint modulating activity. Anti-GITR antibody INCAGN01876 binds to and activates GITRs found on multiple types of T-cells. This stimulates the immune system, induces both the activation and proliferation of tumor-antigen-specific T effector cells (T eff), and suppresses the function of activated T regulatory cells (Tregs). This leads to tumor cell eradication. GITR, a member of the TNF receptor superfamily and $\mathrm{T}$-cell receptor co-stimulator, is expressed on the surface of multiple immune cell types, including Tregs, T effs, B-cells, and natural killer (NK) cells. Inappropriately activated Tregs suppress Teffs and suppress T-cell receptor (TCR) signaling. 